\title{
Nabilone: an alternative antiemetic for cancer chemotherapy
}

\author{
A M DALZELL, H BARTLETT, AND J S LILLEYMAN \\ Department of Haematology, The Children's Hospital, Sheffield
}

SUMMARY A prospective randomised double blind crossover trial was conducted comparing the new synthetic cannabinoid nabilone with oral domperidone in a group of children receiving repeated identical courses of emetogenic chemotherapy for a variety of malignant diseases.

Eighteen of 23 consecutive eligible children, aged 10 months to 17 years, completed the trial. When taking nabilone they experienced significantly fewer vomiting episodes and less nausea, and two thirds expressed a preference for the drug. The most common side effects of treatment with nabilone were somnolence and dizziness, with one patient being disturbed by hallucinations.

The results indicate that nabilone is an effective antiemetic for children having chemotherapy, even for young children. It seems to be superior in this respect to domperidone, and although it has a higher incidence of side effects, these are mostly acceptable to patients. It can be recommended as an alternative to conventional antiemetic treatment throughout childhood.

Vomiting caused by antineoplastic chemotherapy is at best miserable and at worst so disabling and demoralising as to lead to refusal of treatment. There is a variety of antiemetic drugs available to treat the side effect, including the phenothiazines, metoclopramide, and domperidone. Though most of them are partially effective in some patients, none completely solve the problem.

The idea that cannabis might be useful in this context first occurred 10 years ago, ${ }^{1}$ but the side effects and potential for abuse prevented its widespread introduction. Nabilone, a synthetic cannabinoid, was developed to overcome the problems associated with the native drug and has been found to be an effective antiemetic in the field of adult cancer. ${ }^{2} 3$ Its use in children, however, particularly in young children, has yet to be explored. For this reason we conducted a double blind crossover study in a children's cancer unit to compare its performance with domperidone ${ }^{4}$ - generally preferred in the unit at the time as the 'best of the rest'.

\section{Patients and methods}

Consecutive children 17 years old or less undergoing emetogenic antineoplastic chemotherapy for malignant disease were recruited to the study. Full consent for participation was obtained from parents (and patients), and the project was approved by the local ethical committee. To be eligible, patients had to be scheduled to receive two identical courses of emetogenic chemotherapy-identical in terms of drugs, doses, and duration.

The patients were stratified according to weight and received the doses of either nabilone or domperidone shown in Table I. All children on each occasion-that is, each of the two study courses of chemotherapy-received three (or six) identical looking capsules daily or, in the case of some of the very young, three identical looking white powders from broken capsules. The first dose in all cases was taken the night before beginning chemotherapy, and the last dose 24 hours after stopping it. If vomiting was severe enough to prevent effectively oral antiemetic therapy then parenteral (intravenous) domperidone was allowed in addition to the prescribed trial drug. Since the trial was completed this

Table 1 Dosages and regimens of nabilone and domperidone

\begin{tabular}{|c|c|c|}
\hline \multirow{2}{*}{$\begin{array}{l}\text { Weight of } \\
\text { patient }(k g)\end{array}$} & \multicolumn{2}{|l|}{ Regimen } \\
\hline & Nabilone & Domperidone \\
\hline$<18$ & $0.5 \mathrm{mg}$ twice a day & $5 \mathrm{mg}$ three times a day \\
\hline $18-36$ & $1 \mathrm{mg}$ twice a day & $10 \mathrm{mg}$ three times a day \\
\hline$>36$ & $1 \mathrm{mg}$ three times a day & $15 \mathrm{mg}$ three times a day \\
\hline
\end{tabular}


preparation has been withdrawn by the manufacturers.

The trial was a double blind crossover study, nabilone or domperidone being administered with one cycle of chemotherapy and the alternative treatment with the second cycle. The order of drug administration was randomly allocated.

Results were evaluated by patients (where appropriate), their parents, and attendant nursing staff. Patient (parent) questionnaires included details of frequency of actual vomiting (the 'vomit score'), degree of nausea (on a scale of $0-3$ ), and preference for drug A or drug B after completion of the study.

Statistical analysis of the results was carried out using the Wilcoxon matched pairs signed ranks test and McNemar's test with Yates's correction.

\section{Results}

The study took place over a 16 month period and recruited 23 eligible patients. Ten had rhabdomyosarcomas, five had Ewing's tumours, four had acute non-lymphocytic leukaemias, and one each had Hodgkin's disease, a medulloblastoma, a neuroblastoma, and a nasopharyngeal carcinoma. No patients refused to enter the trial, though five were excluded from the analysis. Two patients were withdrawn by their parents because vomiting was considered uncontrolled. Both were on nabilone at the time (cases 19 and 21). Three more entrants did not complete both arms of the crossover. One (case 23) had treatment with nabilone interrupted due to disturbing hallucinations, one (case 22) received two cycles of domperidone in error, and one (case 20) received differing doses of cisplatin on the two cycles. Table 2 shows details of all 23 patients and the drugs they received.

Of the 18 evaluable patients, there were five weighing under $18 \mathrm{~kg}$, nine over 18 but less than 36 $\mathrm{kg}$, and four over $36 \mathrm{~kg}$. Table 3 shows the objective frequency of vomiting in the nabilone and domperidone groups, alongside the subjective (parent/child assessed) nausea score. The frequency of vomiting and nausea scores both show a significant difference in favour of nabilone.

Five of the 18 patients (cases $3,5,14,15$, and 17 on Table 2) required additional intravenous antiemetic treatment for what was considered to be excessive vomiting on both cycles of treatmentthat is, while receiving both nabilone and domperidone. Cases 5 and 15 were the only evaluable children receiving cisplatinum. Five other patients (cases $2,10,11,13$, and 18) also required additional

Table 2 Patient characteristics and drug allocations

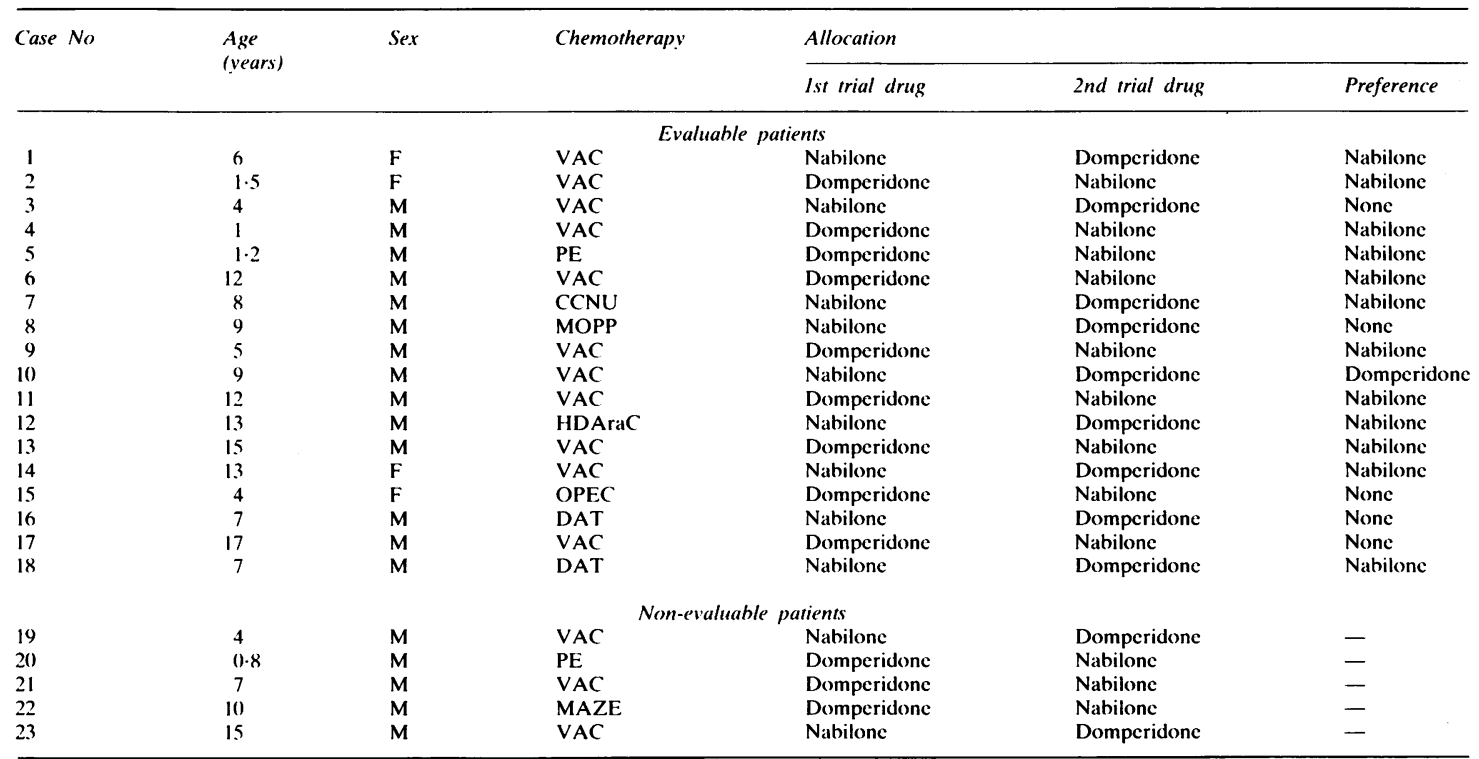

Regimens: VAC=Vincristine, Actinomycin, Cyclophosphamide: PE=Cisplatinum, VP16: MOPP=Mustine. Vincristinc, Procarbazine, Prednisolone: MAZE=M-AMSA. VP16, 5-Azacytidinc: HDAraC=High Dose Cytarabine: OPEC=Vincristine, Cyclophosphamide. Cisplatinum, VM26: DAT= Daunorubican. Cytarabine. Thioguanine. 
Table 3 Efficacy results

\begin{tabular}{|c|c|c|c|c|}
\hline & \multirow{2}{*}{$\begin{array}{l}\text { No of } \\
\text { evaluable } \\
\text { patients }\end{array}$} & \multicolumn{3}{|c|}{ Treatment group } \\
\hline & & Nabilone & Domperidone & p Value \\
\hline Mean No of vomits & 18 & 5.94 & $16 \cdot 72$ & $<0.01+$ \\
\hline Mean severity of nausea* & 18 & $1 \cdot 50$ & $2 \cdot 50$ & $0.01 \dagger$ \\
\hline Preference & 18 & 12 & 1 & $<0 \cdot 01 \ddagger$ \\
\hline
\end{tabular}

* $0=$ None, $1=$ Mild; $2=$ Moderate, $3=$ Severe

+Wilcoxon matched pairs signed rank test.

$\ddagger$ McNemar’s test with Yates’s correction (note: five patients expressed no preference).

parenteral antiemetics, but only on the domperidone arm of the trial. No patient needed extra treatment only on the nabilone arm of the trial.

Twelve patients or their parents, or both, expressed a preference for treatment that turned out to be nabilone, one preferred domperidone, and five did not express a preference (Tables 2 and 3 ). This preference was not influenced by which drug was received first. Nabilone showed a trend towards a better preservation of appetite in some patients, but neither drug completely abolished nausea and vomiting.

Twenty two courses of nabilone and 22 of domperidone were evaluable for toxicity. Adverse side effects were more frequent in the nabilone group, but apart from one patient who hallucinated (he felt his nose was growing while he was shrinking) and withdrew from the trial, these were thought to be acceptable. They are shown in Table 4. Mood changes were only considered adverse if the patient became weepy or depressed. Elevation of mood, which occurred with three patients on nabilone, was regarded as beneficial though was still, strictly speaking, a side effect.

Table 4 Adverse effects to treatment with nabilone or domperidone. Values are No $(\%)$

\begin{tabular}{lll}
\hline & \multicolumn{2}{l}{ Treatment group } \\
\cline { 2 - 3 } & Nabilone & Domperidone \\
\hline No of cycles & 22 & 22 \\
Drowsiness & $12(55)$ & $6(27)$ \\
Dizziness & $8(36)$ & $1(5)$ \\
Mood changes* & $3(14)$ & $1(5)$ \\
Heavy eyed & $2(9)$ & $1(5)$ \\
Pruritus & $1(5)$ & $1(5)$ \\
Dry mouth & $1(5)$ & 0 \\
Vagueness & $1(5)$ & 0 \\
Lightheadedness & $1(5)$ & 0 \\
Increased appetite & $1(5)$ & 0 \\
Hallucinations & $1(5)$ & 0
\end{tabular}

Note: several patients experienced more than one adverse effect. * Mood changes to treatment (a) with nabilonc: depression (one); weeping and clinging to mother (one); crying and hysterical laughter (one); and (b) domperidone: crying and miserable (one).
One subgroup of special interest was the under 2 year olds. There were three such patients evaluable. All of them showed a threefold or greater reduction in the frequency of vomiting while on nabilone, and their parents all indicated a preference for the drug. The fourth infant recruited to the trial (case 20, Table 2) vomited more on the second cycle of treatment (nabilone) but also received a 50\% increase in the dose of cisplatinum. He was therefore excluded from analysis, as noted above.

\section{Discussion}

No universal treatment has yet been found to eliminate the distressing side effect of nausea and vomiting that follows some cancer chemotherapy. Many drugs are partially effective, but it is hard to recommend a 'best buy'. Some patients will prefer one compound while others will favour an alternative. A few will give up in despair and take nothing.

Nabilone represents a new approach to the problem. It is not related in any way to the traditional antiemetics, such as the phenothiazines, metoclopramide, or domperidone, but is a synthetic cannabinoid developed after the earlier observation that marijuana, or $\delta$-9-tetrahydrocannabinol to be precise, seemed able to improve at least the patient tolerance of chemotherapy induced vomiting if not reduce it. ${ }^{1}$

Nabilone shows qualitatively similar central effects to cannabis but has a reduced potential for abuse. ${ }^{6}$ It has been shown to be an effective antiemetic in adult cancer patients and has helped to reduce the severity and incidence of 'chemophobia'-an iatrogenic condition well known to all oncologists, ${ }^{7}$ but documented experience of its use in children has so far been very limited. Two preliminary reports have been made, both of which suggest that it might be better than prochlorperazine. The first was presented to the International Society of Paediatric Oncology in 1983 (unpublished) and the second to the American Society of Clinical Oncology the following year (published only in abstract form). Our study differs from these 
others in two ways. Firstly, it compares the drug with domperidone, arguably a better antiemetic in the context of treatment of cancer than prochlorperazine, at least when given intravenously. ${ }^{45} \mathrm{Sec}$ ondly, it includes young children; up to now nabilone has only been given to children over 5 years of age. Our findings, however, are similar. The cannabinoid was effective in reducing the frequency of vomiting and degree of nausea the patients experienced, and significantly more so than domperidone, despite the small numbers in the study. Equally importantly, most patients or their parents, or both, subjectively preferred nabilone, despite the higher incidence of central side effects.

Of particular interest is the observation that all three of the evaluable patients aged under 2 in our trial vomited less while on nabilone, and their parents thought it the more effective drug. It thus seems to be useful in at least some young children, despite them not having the same tendency to 'chemophobia' as the older children and adolescents. They perhaps provide the best index of the drug's true antiemetic effect.

Although our results come out strongly in its favour, it must be stressed that, like all antiemetic drugs so far available, nabilone certainly does not please all of the patients all of the time. Some it suits well, but some find it ineffective and others find it produces weeping or hallucinations-presumably the equivalent of what would be a 'bad trip' to a marijuana user. Most find its mildly sedative effects not unpleasant, and particularly for some adolescent patients it can turn a five day course of chemotherapy from a dreaded ordeal into something accepted with a shrug of the shoulders. Two such boys in our study had their experience of treatment transformed by the drug to the point where they happily accepted their five days in hospital with a distant look and a stupid grin.

Since the study was completed intravenous domperidone has been withdrawn because of potential toxicity. The consequent loss of what many paediatric oncologists feel to be a particularly effective antiemetic amplifies the need for an alternative, and nabilone seems to be well worth considering.

The authors thank Mr John Timmins, Staff Pharmacist at the Sheffield Children's Hospital, for his help and cooperation and $\mathrm{Dr}$ Marie Bakowski and Ms Sheila Bone of Eli Lilly Limited for their support and help with the study design and analysis.

\section{References}

1 Sallan SE, Zinberg NE, Frei E. Antiemetic effect of delta-9tetrahydrocannabinol in patients receiving cancer chemotherapy. N Engl J Med 1975;293:795-7.

${ }^{2}$ Herman LS, Einhorn LH, Jones SE, et al. Superiority of nabilone over prochlorperazine as an antiemetic in patients receiving cancer chemotherapy. N Engl J Med 1979;300:1295-7.

${ }^{3}$ Einhorn L. Nabilone: an effective antiemetic agent in patients receiving cancer chemotherapy. Cancer Treat Rev 1982;9:55-61.

${ }^{4}$ O'Meara A, Mott MG. Domperidone as an antiemetic in paediatric oncology. Cancer Chemother Pharmacol 1981;6: $147-9$.

s Swann IL, Thompson EN, Qureshi K. Domperidone or metoclopramide in preventing chemotherapeutically induced nausea and vomiting. $\mathrm{Br}$ Med $J$ 1979;ii:1188.

${ }^{6}$ Lemberger L, Rubin A, Wolen R, et al. Pharmacokinetics, metabolism and drug-abuse potential of nabilone. Cancer Treat Rev 1982;9:17-23.

${ }^{7}$ Morrow GR. Prevalence and correlates of anticipatory nausea and vomiting in chemotherapy patients. Journal of the National Cancer Institute 1982;68:585-8.

Correspondence to Dr J S Lilleyman, The Children's Hospital, Sheffield S10 2TH, England.

Received 24 February 1986 